\title{
Perceptual distinctiveness produces long-lasting priming of pop-out
}

\author{
David R. Thomson • Bruce Milliken
}

Published online: 10 January 2012

(C) Psychonomic Society, Inc. 2011

\begin{abstract}
Maljkovic and Nakayama (1994) demonstrated memory influences in singleton search from one trial to the next, an effect they termed priming of pop-out (PoP). This effect was described as resulting from the persistence of an implicit memory trace, the influence of which could be observed for around five to eight subsequent trials. The seemingly short-lived nature of this priming effect has been attributed to decay of the underlying memory representation that occurs when attention is directed to intervening search items, even when such items are perceptually dissimilar from the search trials upon which PoP is measured (Maljkovic \& Nakayama, 2000). The present study reexamines the role of perceptual similarity as a mechanism of interference by examining the influence on PoP of rare search trials that were perceptually distinct with respect to the other, common trials. Long-lasting $(n-16)$ PoP was observed for rare trials that were composed of distinct target/distractor colors, suggesting that $\mathrm{PoP}$ can be observed across at least twice as many trials as has previously been reported. Thus, the time span across which PoP can be measured depends heavily on the nature of the intervening search displays, a result that must be accommodated by current theoretical accounts of PoP.
\end{abstract}

Keywords Priming $\cdot$ Visual search $\cdot$ Memory and attention

Visual search processes are fundamental to the selection of relevant information in our perceptual environment. Although visual search is ubiquitous in everyday life, the nature of the

D. R. Thomson $(\bowtie) \cdot$ B. Milliken

Department of Psychology,

Neuroscience \& Behavior, McMaster University,

1280 Main St. West,

Hamilton, ON L8S 4K1, Canada

e-mail: thomsodr@mcmaster.ca underlying mechanisms that allow us to search efficiently remains a topic of intense scientific study. This study is aimed in particular at the nature of the processes that contribute to efficient (or pop-out) search performance. Although pop-out search was once thought of as depending entirely on preattentive perceptual processing (Treisman \& Gelade 1980), a growing body of evidence suggests that it is also affected by shortterm memory representations. Assessing the seemingly shortlived nature of memory representations in singleton search was the primary purpose of the work reported here.

In a series of experiments, using a task first developed by Bravo and Nakayama (1992), Maljkovic and Nakayama $(1994,1996,2000)$ systematically examined the role of memory in singleton search (see also Treisman, 1992, for a description of intertrial repetition effects in feature search). In their experiments, participants searched for an oddcolored diamond among two homogeneous distractors and indicated whether it was truncated on the left or the right. Performance was analyzed on the current trial as a function of the repetition or alternation of the target-defining feature from the previous trial. Importantly, they found that search times were shorter for repeated target colors, as compared with switches, an effect they termed priming of pop-out (PoP). PoP, it was argued, reflects the operation of a shortterm, implicit memory trace that contains target-defining feature information. This memory trace was shown to affect performance for five to eight subsequent trials (Maljkovic \& Nakayama, 1994, Experiment 5) and was also shown to affect performance without awareness (Maljkovic \& Nakayama, 2000). The decay of the memory trace over the five to eight trials was argued to be "gradual," perhaps following an exponential decay function.

Although the gradual decline in the PoP effect across five to eight trials could be accounted for by a passive decay process, Maljkovic and Nakayama (2000) noted a possible 
role for interference from intervening items in PoP. In particular, they suggested that shifts of attention to the target on trials intervening between the influencing trial (on trial $n-i$ ) and the current trial (trial $n$ ) reduce the strength of the memory trace underlying PoP. Furthermore, they noted that these intervening items need not be perceptually similar to the actual search trials to produce the gradual decline in PoP effects with intervening items.

Perhaps because similarity-based interference was regarded as unnecessary to explain the decline in PoP across intervening trials, $\mathrm{PoP}$ is now often explained by reference to activation/suppression processes. This account assumes that when pop-out search is performed, abstract feature representations that define the target are modified. For example, if the task is to locate the odd-colored item in a display and it happens to be green, while distractors are red, a corresponding feature gain for "green" and feature suppression for "red" will occur. These feature activation changes then begin to return back to baseline, postresponse, such that if they are still activated or suppressed when the next search array appears, costs or benefits to performance can be observed (Goolsby \& Suzuki, 2001; Lamy, Antebi, Aviani, \& Carmel, 2008; Lee, Mozer, \& Vecera, 2009).

An alternative theoretical account of PoP relies on memory retrieval processes. For example, some researchers have found that priming effects in singleton search from one trial to the next can depend not only on the repetition/alternation of the target-defining feature, but also on target-irrelevant feature repetitions, such as the repetition/alternation of the response feature (Hillstrom, 2000), irrelevant spatial frequency (Kristjánsson, 2006), and irrelevant color in an odd-sized pop-out task (Huang, Holcombe, \& Pashler, 2004). The fact that repeating or alternating these low-level features from one trial to the next produces interactions in performance (sometimes in line with partial-match effects; see Hommel, 1998, 2004) has led to the conclusion that perhaps PoP is driven by the implicit and automatic retrieval of bound episodic memory representations (Hillstrom, 2000; Huang et al., 2004; Thomson \& Milliken, 2011), akin to tokens (Treisman, 1992), instances (Logan 1988, 1990), or event files (Hommel, 1998). Hillstrom (2000) argued that if these episodic representations drive PoP, they are likely stored temporarily in visual short-term working memory (VSWM) on a given trial and are subsequently recruited from VSWM on the next trial, on the basis of some "preattentive" map of the current display that shares low-level perceptual attributes with the episode stored in VSWM. In summary, in contrast to feature gain modulations that carry forward in time, episodic representations are retrieved from memory by the perceptual attributes of the current search display.

Rather than aligning with one or the other of these two explanatory frameworks, some researchers have suggested recently that PoP may be the result of both activation/suppression and episodic retrieval processes. In particular, it has been suggested that facilitatory effects observed in intertrial priming may be an "aggregate" of early perceptual-processing benefits in directing focal attention to the odd-ball target, in addition to later memory retrieval benefits that impact response-related processing (Lamy, Yashar, \& Ruderman, 2010). An important implication of this hybrid view is that recent studies showing that episodic retrieval can impact singleton search performance from one trial to the next does not preclude the presence of early perceptual-processing benefits that are due to activation/ suppression mechanisms. Although the details of when one or the other process is responsible for PoP effects require additional study, it has also been suggested that episodic influences tend to be expressed in performance when search stimuli can be perceived as integrated objects (Kristjánsson, Ingvarsdóttir, \& Teitsdóttir, 2008) or when target-distractor discriminability is low, thus making search less efficient (Asgeirsson \& Kristjánsson 2011; Lamy, Zivony, \& Yashar, 2011; Yashar \& Lamy, 2011).

Despite the important differences between current episodic retrieval, activation/suppression, and hybrid accounts of PoP, central to all accounts is the idea that priming in singleton search is a relatively short-lived phenomenon. For example, as was mentioned earlier, Maljkovic and Nakayama (1994, Experiment 5) demonstrated that the memory trace laid down on a given search trial could exert its influence for up to five to eight subsequent trials (Geyer, Muller, \& Krummenacher, 2007, found PoP for location that lasted only four trials; Hillstrom, 2000, found PoP for color that lasted only three trials). Feature gains, therefore, are argued to "decay" within a few trials, whereas episodes can be retrieved only while they are in VSWM. In effect, there appears to be a consensus among proponents of both activation/suppression accounts and episodic retrieval accounts of PoP that long-term episodic memory representations known to impact performance in less efficient (conjunctive) search scenarios (see Chun \& Jiang, 1998; Chun \& Phelps, 1999) do not contribute to PoP.

Indeed, theoretical accounts of priming effects in human performance tasks have often been constrained by an assumption that they are driven by short-term memory representations. For example, inhibition of return (IOR; see Posner \& Cohen, 1984) was long assumed to reflect the temporary "suppression" of attention to a previously attended location. However, Tipper, Grison, and Kessler (2003) found IOR effects that survived a number of intervening trials, when complex displays (faces) were used. Similarly, Wilson, Castel, and Pratt (2006) found long-term IOR effects by presenting "rare" IOR trials that appeared in different spatial locations than the intervening "common" trials. Such effects have been taken as evidence for the role of memory retrieval processes in producing IOR that can be long term in nature. Following this line of thinking, the present study examined whether perceptually distinct rare trials in a color-singleton search task might 
produce long-term PoP effects - that is, PoP effects that survive more than five to eight intervening trials. Such a finding would have significant theoretical implications for current accounts of the memory mechanism underlying PoP-in particular, indicating that at least one of the determinants of the PoP effect is indeed sensitive to similarity-based interference.

\section{The present study}

In order to assess whether PoP can be observed across a large number of intervening items, rare search arrays were created that were perceptually distinct with respect to the intervening common trials. Thus, participants performed a color pop-out search task in which common trials were composed of one target/distractor color set (e.g., red/green or blue/yellow), while rare trials (which appeared every 16th trial) were composed of the other target/distractor color set. Although we expected priming on the standard common trials to last for about five to eight subsequent trials, as has been shown previously, we hypothesized that rare trials may constitute perceptually distinct contexts for which longerlasting PoP may be observed.

\section{Method}

Participants Twenty-four undergraduate students from McMaster University (11 male, 13 female; mean age = 20.8 years) participated in exchange for either course credit or $\$ 10 \mathrm{CAD}$ for $1 \mathrm{~h}$ of participation. All participants had normal or corrected-to-normal vision.

Apparatus/stimuli Search arrays were presented using Presentation software on a Dell 15-in. CRT monitor connected to a Dell PC. Each search array contained three squares that were separated from one another at a distance of $1.0^{\circ}$ of visual angle and that subtended a vertical and horizontal visual angle of $1.1^{\circ}$ each. Each square contained a gap in either the left or right side that subtended a visual angle of $0.6^{\circ}$ vertically. Each search array was arranged in an equilateral triangle with a fixation cross at the center, which subtended a vertical and horizontal visual angle of $0.6^{\circ}$. Each array contained two homogeneous distractor items and one target item (i.e., if the target item was randomly assigned to be red on a given trial, the distractors were presented in green). Common trials were composed of one target-distractor color set (e.g., red/green or blue/yellow), while rare trials were composed of the other target-distractor color set. This assignment of color sets to common and rare trials was counterbalanced across participants.

Procedure Participants were seated approximately $57 \mathrm{~cm}$ from the computer screen and were instructed that, on each trial, they would see three squares arranged in a triangular formation around a central fixation cross. They were asked to locate the odd-colored square in each display and indicate whether it had a gap in the left or right side as quickly and accurately as possible. Participants indicated a left response by hitting the " $z$ " key with their left index finger and indicated a right response by hitting the "/ key with their right index finger.

Each stimulus array was preceded by a fixation cross that appeared $300 \mathrm{~ms}$ before the search array and remained on the screen until a response was made, at which point the stimulus array, together with the fixation cross, disappeared. The screen then remained blank for $500 \mathrm{~ms}$ until the fixation cross appeared again to signal the next trial. This resulted in an 800-ms response-to-stimulus interval between search arrays. Participants completed 15 search trials composed of one target/distractor color set (red/green or blue/yellow) and then completed 1 search trial composed of the other target/distractor color set. ${ }^{1}$ This sequence was repeated 100 times for a total of 1,600 trials (1,500 common, 100 rare). The first 8 trials in the experiment were used for practice and instructional purposes, resulting in 1,592 experimental trials. Participants were given the opportunity to take a break every 200 trials, and the experiment resumed whenever the participants pressed the space bar to initiate the next block. The trial sequence is presented in Fig. 1a.

\section{Results}

Only trials on which correct responses were made were submitted to the response time (RT) analysis. Since each experimental condition was contingent on the nature of the previous trial type, RTs and errors for trials immediately following an error response were also excluded from analysis. Following this logic, in terms of our analysis of priming effects on trial $n$ as a function of trial $n-i$, RTs and errors on trial $n$ following an error response on trial $n-i$, were aslo excluded. All remaining RTs were submitted to an outlier procedure that excluded RTs based on outlier criteria that varied as a function of cell size (see Van Selst \& Jolicoeur, 1994). Separate cells of observations were created using RTs for common trials as a function of whether the target color repeated or switched in relation to the immediately preceding trial $(n-1)$, to the trial

\footnotetext{
${ }^{1}$ Although this design allows for the possibility that participants might predict when a rare trial would occur, there was no way for them to know whether the target color would be a repetition or a switch from the previous rare trial. If anything, the predictable nature of the design may have diminished the costs associated with responding to a rare trial. In any case, no participants reported using an idiosyncratic strategy in which they kept in mind the target color for the rare trials for the purpose of predicting the target color for a subsequent rare trial, and we regard this hypothesis as an very unlikely candidate explanation for the results reported here.
} 


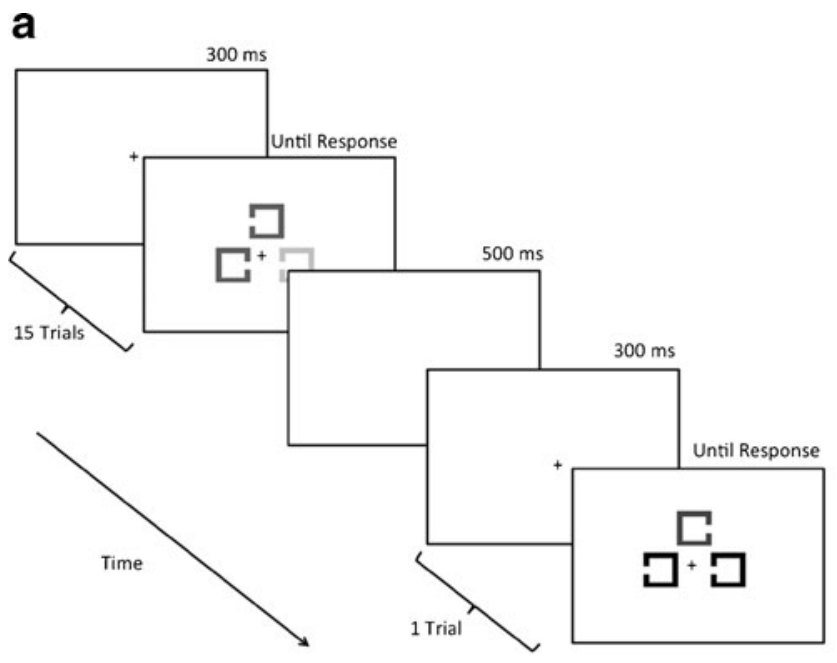

b

Reaction Time as a Function of Influencing Trial

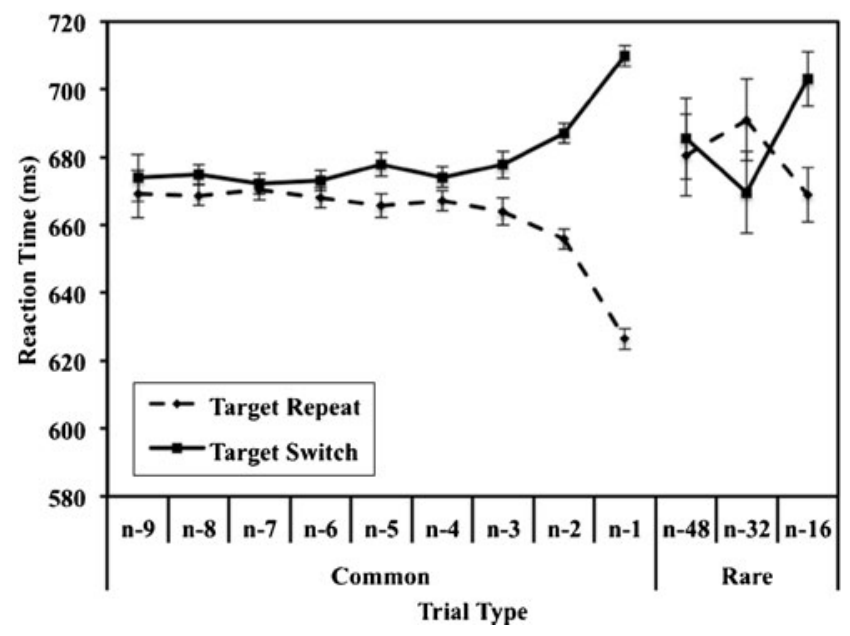

Fig. 1 a A depiction of the procedure used in the present study, in which participants locate and respond to the odd-colored square in each display. Stimulus displays remained on the screen until a response was executed, and then, after a 500-ms blank interval, the fixation cross for the next search array appeared. Participants performed 15 common trials, followed by 1 rare trial. b Response times (RTs; in milliseconds) on trial $n$ are depicted for target repetitions and switches and for common and rare trial types as a function of influencing trial $(n-i)$. Error bars depict the standard error of the priming effects (target switch RT - target repeat RT)

just before the immediately preceding trial $(n-2)$, and so on, back to trial $n-9$. Note that this analytic strategy implied that any particular RT could contribute to the mean RT for multiple preceding trial types (e.g., trial $n$ RT might contribute to the target repeat mean RT for the $n-1$ preceding trial type but to either the target repeat or target switch mean RT for the $n-2$ preceding trial type). Similarly, separate cells of observations were created using RTs for rare trials as a function of whether the target color repeated or switched from the immediately preceding rare trial $(n-16)$, as well as prior influencing rare trials $(n-32$ and $n-48)$. Overall, the outlier analysis eliminated $2.7 \%$ of the observations. Mean RTs were then computed from the remaining observations. These mean RTs are shown in Fig. 1b. Error rates were computed in a similar manner for first-order sequence effects $(n-1$ for common trials, $n-16$ for rare trials). A summary of error rates for target color repetitions and switches on common trials as a function of the immediately preceding common trial $(n-1)$ and on rare trials as a function of the immediately preceding rare trial $(n-16)$ are shown in Table 1.

Our analyses centered on three issues. First, we compared performance for the common and rare trials, simply to evaluate whether this factor affected performance overall. Second, we examined performance on common trials, excluding those trials on which the preceding trial was a rare trial. Our objective here was to evaluate whether the data replicate the results reported in prior studies that have not included rare trials. Third, and most important, we examined performance on rare trials to evaluate whether PoP effects can occur relative to other rare trials that happened 16 trials prior.

Response times There was a significant difference between RTs for common and rare trials, $t(23)=2.58, d=0.17$, $p=.0166$, with responses being faster on common trials (668 ms) than on rare trials $(686 \mathrm{~ms})$. To evaluate the magnitude of the PoP effect for common trials as a function of the previous influencing common trials, planned comparisons were conducted that compared target repetitions and alternations. These analyses indicated significant PoP effects on the current trial as a function of the following influencing trials: $n-1$ (83 ms), $t(23)=11.87, d=0.81, p<.0001 ; n-2$ $(31 \mathrm{~ms}), t(23)=9.90, d=0.30, p<.0001 ; n-3(14 \mathrm{~ms}), t(23)=$ 4.76, $d=0.13, p<.0001 ; n-4(7 \mathrm{~ms}), t(23)=2.50, d=$ $0.07, p=.0201 ; n-5(12 \mathrm{~ms}), t(23)=3.65, d=0.12, p=$ .0013 ; and $n-8(6 \mathrm{~ms}), t(23)=2.25, d=0.06, p=.0341$; there were no significant PoP effects as a function of the following influencing trials: $n-6(5 \mathrm{~ms}), n-7(2 \mathrm{~ms})$, and $n-9(5 \mathrm{~ms})$. These results mirror those of prior studies in which the target color on the current trial has been shown to affect performance for about five to eight subsequent trials (Maljkovic \& Nakayama, 1994).

Table 1 Mean error rates $(M \mathrm{~s})$ and standard deviations $(S D \mathrm{~s})$ in percentages, for target color repetitions and alternations as a function of trial type

\begin{tabular}{|c|c|c|c|c|}
\hline & \multicolumn{4}{|c|}{ Trial Type } \\
\hline & \multicolumn{2}{|c|}{ Common } & \multicolumn{2}{|c|}{ Rare } \\
\hline & $M$ & $S D$ & $M$ & $S D$ \\
\hline Target repeat & 3.8 & 3.3 & 3.5 & 3.7 \\
\hline Target switch & 5.3 & 4.1 & 2.9 & 3.1 \\
\hline
\end{tabular}


Of primary interest to the present study is whether PoP can be observed from one rare trial to the next, across 15 intervening common trials. To assess the magnitude of the PoP effect on the current rare trial as a function of the previous influencing rare trials, planned comparisons were conducted that compared target repetitions and alternations. These analyses revealed a significant PoP effect at $n-16(34 \mathrm{~ms}), t(23)=2.92, d=0.29, p=$ .0077 , but not at $n-32(-21 \mathrm{~ms})$ or $n-48(5 \mathrm{~ms})$. This result indicates that although PoP for common trials diminished following an interval containing five to eight common trials, PoP was still measurable from one rare trial to the next following an interval between the 2 rare trials that contained 15 common trials.

Error rates The analysis of error rates revealed that significantly more errors were committed on common trials $(4.5 \%)$ than on rare trials $(3.2 \%), t(23)=3.11, d=0.41, p=.0049$, suggesting perhaps that the onset of a rare trial led participants to respond both more slowly and more accurately overall. In the analysis of the common trials, more errors were committed on target switches (5.3\%) than on target repetitions (3.8\%), $t$ $(23)=4.65, d=0.39, p<.0001$, relative to the immediately preceding trial $(n-1)$. In the analysis of the rare trials, there was no significant difference in error rates for target repetitions $(3.5 \%)$ and switches $(2.9 \%)$, relative to the immediately preceding rare trial $(n-16) .^{2}$

\section{Discussion}

The primary purpose of the present study was to examine whether perceptually distinct search arrays in a color pop-out task would yield priming of pop-out across a large number of intervening items. PoP was originally shown to last for about five to eight trials (Maljkovic \& Nakayama, 1994, Experiment 5) and was, therefore, classified as a short-term influence of memory on behavior. The results reported here constitute the first demonstration of longer-lasting PoP for particular target/ distractor colors after only a single stimulus exposure.

On the basis of the short-term nature of PoP, theoretical interpretations of the memory mechanism underlying PoP have posited that feature gains associated with targetdefining features decay within five to eight trials, on the one hand (Lee et al., 2009; Maljkovic \& Nakayama, 1994), or that bound episodic representations are retrieved from a short-term memory store, on the other hand (Hillstrom,

\footnotetext{
2 The reader may note that although there is no statistical difference in error rates between target repetitions and switches for rare trials, numerically the difference is opposite to that of RTs. The observation of PoP from one rare trial to the next has been replicated twice in our lab (unpublished data), and in no case was there a statistical difference in error rates between target color repetitions and switches. In fact, pooling data from the three experiments in which we have observed long-lasting PoP, the error rates for target repetitions $(3.9 \%)$ and switches (3.5\%) are nearly identical, $t(71)=0.85, d=0.09, p=.399$.
}

2000; see also Huang et al., 2004). The work reported here follows the logic of similar studies that have sought longerterm priming effects in other attention and performance domains (e.g., IOR; see Wilson et al., 2006). In particular, the aim of the present study was to examine whether perceptually distinct rare trials would allow for context-specific retrieval processes that produce PoP effects across a relatively large number of intervening search experiences.

The results were quite clear, in that PoP effects were shown to persist for 5-8 trials for the common search arrays, which parallels the findings from other singleton search studies. Importantly, though, significant PoP emerged when measured from one rare trial to the next in the present study, despite the fact that such an effect was measured across 15 intervening search trials. PoP, therefore, can be said to last for at least 16 trials, provided that trial $n$ and trial $n-i$ are perceptually distinct with respect to the intervening search arrays. This result suggests that the perceptual similarity of intervening search displays has a significant impact on the time course across which the memory representations underlying PoP operate.

Indeed, our result converges with other recent work suggesting that there may actually be multiple time courses on which the memory trace driving PoP operates. For example, Brascamp, Pels, and Kristjánsson (2011) demonstrated that when participants were exposed to a large sequence of target repetitions in a color pop-out task, priming effects for that target survived longer than priming effects that followed from only one or two repetitions of a target color. It was therefore argued that the memory trace for a given target color can decay quickly or slowly, depending on recent trial history over a number of experiences (see also Martini, 2010, for additional evidence that target-defining feature information may decay at different rates). In effect, these authors are arguing that the PoP effect can last longer than previous demonstrations of the time course of PoP because of particularly slow decay processes associated with particular target/distractor colors. It might well be the case that our result also reflects slower decay processes associated with the memory trace underlying PoP, but when tirals that intervene between the current and influencing trials are dissimilar. Alternatively, it may be that our result does not implicate decay processes at all but, rather, that our manipulation of perceptual similarity actually served to reduce retroactive interference effects associated with the intervening trials (the common trials), such that a perceptually distinct rare trial uniquely cued the retrieval of the most recent rare trial memory representation. The present data do not allow us to differentiate between these two theoretical interpretations of the longer-lasting $(n-16)$ PoP effects we have observed but do converge with other recent work to support the idea that the time course over which memory representations underlying PoP operate can be quite long.

In summary, the novel finding of long-lasting PoP observed here has important implications for the theoretical 
accounts of PoP offered in the introduction. In light of the present results, an account of PoP that hinges on activation/ suppression of feature gains from one trial to the next must concede that feature gains decay at a much slower rate for the rare than for the common trial type. This may not be problematic for such accounts, especially since strict limits on the time course of such feature-gain decay processes have not been posited. In particular, Maljkovic and Nakayama (2000) argued that the decay of the memory trace underlying PoP lasts at least $30 \mathrm{~s}$, but not more than minutes. An account of PoP that hinges on the retrieval of bound episodic representations, on the other hand, must concede that these representations are likely not recruited from VSWM (assuming that 16 search experiences is well beyond the capacity limitations of such a store). In either case, the important point is that long-lasting PoP effects delivered by use of perceptually distinct displays provide an empirical tool with which to examine the temporal limits of the memory representation underlying PoP. In addition, these results suggest that the nature of interference from intervening search experiences between trial $n-i$ and trial $n$ has a significant impact on the "duration" of PoP.

Clearly, a great deal more work is necessary to explore what types of perceptual/contextual distinctiveness produce the long-lasting PoP observed here. Specifically, the $n-16$ PoP for rare search displays could be explained with reference to either slow-decaying feature gains (as in an activation/suppression account) or context-specific retrieval processes (as in an episodic retrieval account). In order to differentiate between these two explanations for long-lasting PoP, further work is needed that manipulates contextual dimensions of the rare search displays, other than target/ distractor colors (upon which feature gains presumably operate). For example, if rare search displays were differentiated from common search displays along some higherlevel contextual dimension (but were otherwise perceptually identical) and rare trial PoP effects persisted, context-specific retrieval processes may be implicated over slow-decaying feature gains as the most likely explanation for the long-lasting PoP observed here. Nonetheless, the present experiment on its own makes the point that PoP can be measured across intervals that are at least twice as long as has previously been shown and provides new evidence for the role of interference as a primary contributor to the short-term nature of PoP that has been observed in other work.

Acknowledgement This research was funded by an NSERC Discovery Grant awarded to B.M. We would also like to thank two anonymous reviewers for their helpful comments on an earlier version of the manuscript, as well as Afrisa Yeung for help with data collection.

\section{References}

Asgeirsson, A. G., \& Kristjánsson, A. (2011). Episodic retrieval and feature facilitation in intertrial priming of visual search. Attention, Perception, \& Psychophysics, 73, 1350-1360.

Brascamp, J. W., Pels, E., \& Kristjánsson, A. (2011). Priming of popout on multiple time scales during visual search. Vision Research, 51, 1972-1978.

Bravo, M. J., \& Nakayama, K. (1992). The role of attention in different visual-search tasks. Perception \& Psychophysics, 51, 465-472.

Chun, M. M., \& Jiang, Y. (1998). Contextual cueing: Implicit learning and memory of visual contest guides spatial attention. Cognitive Psychology, 36, 28-71.

Chun, M. M., \& Phelps, E. A. (1999). Memory deficits for implicit contextual information in amnesiac subjects with hippocampal deficits. Nature Neuroscience, 2, 844-847.

Geyer, T., Muller, H. J., \& Krummenacher, J. (2007). Cross-trial priming of element positions in visual pop-out search is dependent on stimulus arrangement. Journal of Experimental Psychology: Human Perception and Performance, 33, 788-797.

Goolsby, B. A., \& Suzuki, S. (2001). Understanding priming of colorsingleton search: Roles of attention at encoding and retrieval. Perception \& Psychophysics, 63, 929-944.

Hillstrom, A. P. (2000). Repetition effects in visual search. Perception \& Psychophysics, 62, 800-817.

Hommel, B. (1998). Event files: Evidence for automatic integration of stimulus-response episodes. Visual Cognition, 5, 183-216.

Hommel, B. (2004). Event files: Feature binding in and across perception and action. Trends in Cognitive Sciences, 8, 494-500.

Huang, L., Holcombe, A. O., \& Pashler, H. (2004). Repetition priming in visual search: Episodic retrieval, not feature priming. Memory \& Cognition, 32, 12-20.

Kristjánsson, A. (2006). Simultaneous priming along multiple feature dimensions in a visual search task. Vision Research, 46, 2554-2570.

Kristjánsson, A., Ingvarsdottir, A., \& Teitsdottir, D. (2008). Object-and feature-based priming in visual search. Psychonomic Bulletin \& Review, 15, 378-384.

Lamy, D., Antebi, C., Aviani, N., \& Carmel, T. (2008). Priming of popout provides reliable measures of target activation and distracter inhibition in selective attention. Vision Research, 48, 30-41.

Lamy, D., Yashar, A., \& Ruderman, L. (2010). A dual-stage account of inter-trial priming effects. Vision Research, 50, 1396-1401.

Lamy, D., Zivony, A., \& Yashar, A. (2011). The role of search difficulty in intertrial feature priming. Vision Research, 51, 20992109. doi:10.1016/j.visres.2011.07.010

Lee, H., Mozer, M. C., \& Vecera, S. P. (2009). Mechanisms of priming of pop-out: Stored representations or feature-gain modulations? Attention, Perception, \& Psychophysics, 71, 1059-1071.

Logan, G. D. (1988). Toward an instance theory of automatization. Psychological Review, 95, 492-527.

Logan, G. D. (1990). Repetition priming and automaticity: Common underlying mechanisms? Cognitive Psychology, 22, 1-35.

Maljkovic, V., \& Nakayama, K. (1994). Priming of pop-out: I. Role of features. Memory \& Cognition, 22, 657-672.

Maljkovic, V., \& Nakayama, K. (1996). Priming of pop-out: II. The role of position. Perception \& Psychophysics, 58, 977-991.

Maljkovic, V., \& Nakayama, K. (2000). Priming of pop-out: III. A short-term implicit memory system beneficial for rapid target selection. Visual Cognition, 7, 571-595.

Martini, P. (2010). System identification in priming of pop-out. Vision Research, 50, 2110-2115.

Posner, M. I., \& Cohen, Y. A. (1984). Components of visual orienting. In H. Bouman \& D. G. Bouwhuis (Eds.), Attention and performance $X$ (pp. 531-556). Hove: Erlbaum. 
Thomson, D. R., \& Milliken, B. (2011). A switch in task affects priming of pop-out: Evidence for the role of episodes. Attention, Perception, \& Psychophysics, 73, 318-333.

Tipper, S. P., Grison, S., \& Kessler, K. (2003). Long-term inhibition of return of attention. Psychological Science, 14, 1925.

Treisman, A. M., \& Gelade, G. (1980). A feature-integration theory of attention. Cognitive Psychology, 12, 97-136.

Treisman, A. (1992). Perceiving and re-perceiving objects. American Psychologist, 47, 862-875.
Van Selst, M., \& Jolicoeur, P. (1994). A solution to the effect of sample size on outlier elimination. Quarterly Journal of Experimental Psychology, 47, 631-650.

Wilson, D. E., Castel, A. D., \& Pratt, J. (2006). Long-term inhibition of return for spatial locations: Evidence for a memory retrieval account. Quarterly Journal of Experimental Psychology, 59, 2135-2147.

Yashar, A. \& Lamy, D. (2011). Refining the dual-stage account of intertrial feature priming: Does motor response or response feature matter? Attention, Perception, \& Psychophysics, 73, 2160 2167. 